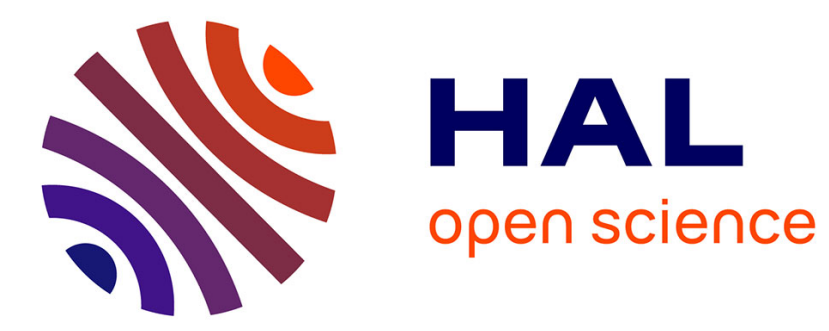

\title{
Electric dipolar echoes in smoky quartz
}

L. Bernard, M. Saint-Paul, J. Joffrin

\section{- To cite this version:}

L. Bernard, M. Saint-Paul, J. Joffrin. Electric dipolar echoes in smoky quartz. Journal de Physique Lettres, 1979, 40 (22), pp.593-597. 10.1051/jphyslet:019790040022059300 . jpa-00231695

\section{HAL Id: jpa-00231695 https://hal.science/jpa-00231695}

Submitted on 1 Jan 1979

HAL is a multi-disciplinary open access archive for the deposit and dissemination of scientific research documents, whether they are published or not. The documents may come from teaching and research institutions in France or abroad, or from public or private research centers.
L'archive ouverte pluridisciplinaire HAL, est destinée au dépôt et à la diffusion de documents scientifiques de niveau recherche, publiés ou non, émanant des établissements d'enseignement et de recherche français ou étrangers, des laboratoires publics ou privés. 


\title{
Electric dipolar echoes in smoky quartz
}

\author{
L. Bernard, M. Saint-Paul \\ C.R.T.B.T., C.N.R.S., B.P. 166, 38042 Grenoble Cedex, France
}

and J. Joffrin $\left(^{*}\right)$

$\left(^{*}\right)$ Institut Laue-Langevin, $156 \mathrm{X}, 38042$ Grenoble Cedex, France

(Reçu le 26 juillet 1979, révisé le 4 septembre 1979, accepté le 13 septembre 1979)

\begin{abstract}
Résumé. - On observe des échos dipolaires électriques dans un cristal de quartz naturel, irradié par rayonnement gamma. Ces expériences sont réalisées dans la gamme de température 5 à $20 \mathrm{mK}$ et à une fréquence de $360 \mathrm{MHz}$. L'étude de la dépendance des échos spontané et stimulé en fonction de l'amplitude des impulsions ainsi que leur décroissance en fonction du temps indique qu'il existe deux types de dipoles : on évalue leurs moments transverses et longitudinaux puis leur densité d'état.
\end{abstract}

Abstract. - Electric dipolar echoes are observed in gamma irradiated natural quartz crystal in the 5 to $20 \mathrm{mK}$ range at a frequency of $360 \mathrm{MHz}$.

Studying the behaviour of both spontaneous and stimulated echoes, their dependance on the input pulse amplitude and their decay in time, we are led to think that there are two kinds of dipoles ; their transverse and longitudinal electric moments are evaluated ; their density of state is also extracted.

1. Introduction. - The anomalous low temperature properties of $\gamma$-irradiated quartz have been studied by different techniques for a long time. The observed anomalies have been attributed to holes trapped at aluminium substitutions impurities [1]. Several arguments suggest that in smoky quartz a hole, that is an electron defect, is trapped at one of the four nearest neighbour oxygen of that aluminium impurity; that hole can move between two equivalent oxygen sites and generate consequently a two-level system which seems to be responsible for many of these anomalous properties. Electronic dipole resonance [2] at a frequency of $9 \mathrm{GHz}$ is due to a resonant transition between the two configurational states which are stark-splitted by the cristalline field or by the tunneling between these two states. The distribution in energy of these two-level systems (TLS) is rather broad, of the order of $10^{-16} \mathrm{erg}$. In that original paper, the analogy with a case of ionic dipole resonance was already remarked and it was also outlined that there was a strong coupling of these centres with the lattice. Later on, an extensive study of the dielectric properties of smoky quartz was done [3]; assuming a gaussian distribution of the properties of these TLS, it was possible to explain both the real part, the imaginary part of the dielectric constant, and the influence of a static electric field superimposed upon the alternating measuring field; in particular, in the temperature range of $4 \mathrm{~K}$, the characteristic frequency of the dielectric constant was of the order of $1 \mathrm{kHz}$. This is in fact characteristic of a rather poorly coupled system with the lattice. More recently, the authors having in mind the analogy with silica glass, the different features of smoky quartz were reviewed. The specific heat of smoky quartz [4] was proved to have a linear temperature dependence below $1 \mathrm{~K}$; the high frequency ultrasonic relaxation was related with a Debye relaxation peak at low and high temperature [5] and finally the thermal conductivity down to $30 \mathrm{mK}$ [6] was proven to be unchanged in comparison with the non-irradiated quartz.

In this letter, we report new results on the electrical properties of these TLS using a technique which has already been used in glasses [7] to explore both the distribution of properties of polarizable centres and the different relaxation times at very low temperatures. In order to study the dynamic properties of these centres in quartz, we make use of the fact that they are easily coupled to an external electric field : we apply a sequence of two or three electrical R.F. pulses 
to a thin disk of gamma irradiated quartz crystal. There is a resonant interaction with those TLS that have an energy splitting that lies within the bandwidth of the electrical pulse. The population of these defects is coherently excited by the first pulse. Because these TLS interact with their environment (thermal phonons, other defects) this state evolves in a partly coherent and partly incoherent way. A second pulse applied after a time $\tau$ restores an echo at time $2 \tau$ whose amplitude is governed only by the irreversible evolution of the system : we observe a spontaneous dipolar electrical echo. When a third pulse is applied to the system, at a time $t$ after the second one, it generates a new kind of echo : a stimulated echo which appears at time $t+2 \tau$ and whose amplitude is governed not only by the coherence time but also by the relaxation time $T_{1}$ of the population of the excited TLS. We have systematically measured the amplitude of these echoes as function of the incident power, of the temperature and of the times $\tau$ and $t$.

2. Experimental set-up. - The quartz crystal sample [12] consists of a disk $(10 \mathrm{~mm}$ in diameter, $2 \mathrm{~mm}$ in thickness) to which superconducting electrodes are bonded. In order to avoid any phonon echo or piezoelectric coupling, the disk is cut perpen-

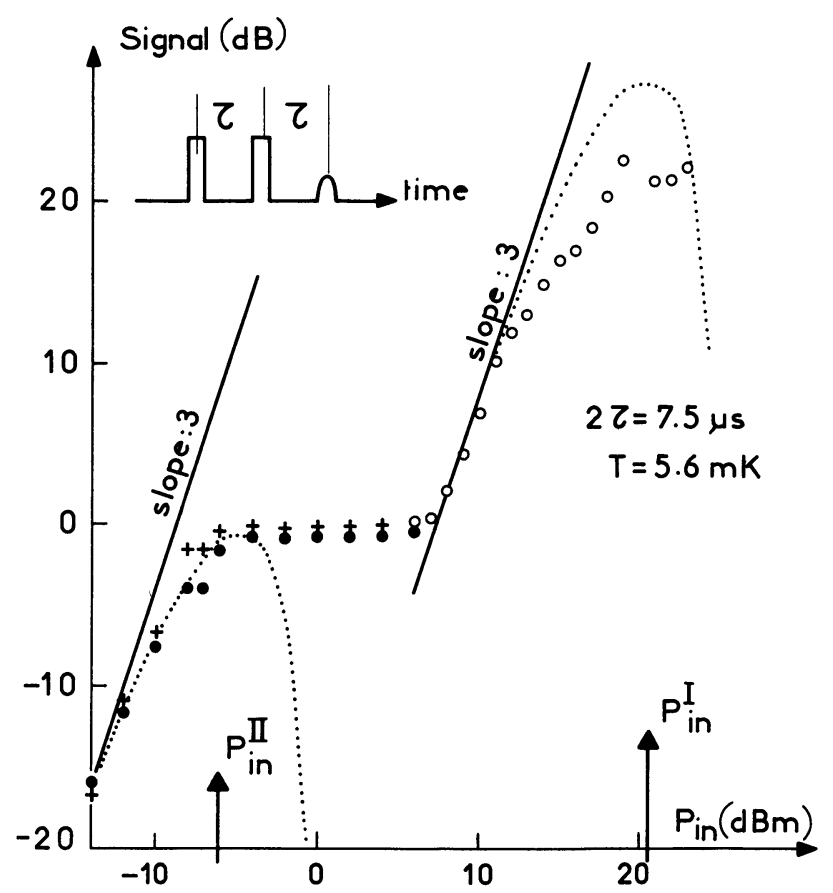

Fig. 1. - Spontaneous signal in $\mathrm{dB}$ as a function of the incident power (in $\mathrm{dBm}$ ). To obtain the true power applied onto the crystal $5 \mathrm{~dB}$ corresponding to the losses on the line must be substracted. According to the same effect the calculated echo signal must be increased by $5 \mathrm{~dB}$. Note the initial slope equal to three for the two types of centres. The crosses correspond to measurements with an integrator of the echo signal ; the black points correspond to the measurement with a box-car centered onto the top of the echo : the open circles correspond to the one shot measurement with an integrator. The dotted curve result from a theoretical calculation of the signal corresponding to a $(\pi / 2, \pi)$ pulses applied to a narrow distribution of dipole. dicular to the $C$ axis out of a single crystal of natural quartz. The sample has been irradiated at room temperature with a ${ }^{60}$ Cobalt source; the irradiation dose was $10^{7}$ rads. The impurity content is mainly aluminium with a concentration of $37 \mathrm{ppm}$. The same sample was previously used for specific heat [4], dielectric constant and thermal conductivity [6] studies.

The disk make up the capacitor $(2 \mathrm{pF})$ of a resonant circuit tuned at $360 \mathrm{MHz}$ and matched to a $50 \Omega$ coaxial line. At low temperature, the electrical quality factor $Q$ of the circuit is 250 . The ensemble is immersed in the mixing chamber of a dilution refrigerator; the cryogenic characteristics have been previously described and are similar to the experiment performed in publications [7, 11].

The peak pulse power sent to the sample ranges from $-16 \mathrm{dBm}$ to $+20 \mathrm{dBm}$. The detector is sensitive to roughly $-100 \mathrm{dBm}$ and is calibrated such that $0 \mathrm{~dB}$ corresponds to a signal of $-73 \mathrm{dBm} \pm 1$. The insertion loss of the coaxial line is $5 \mathrm{~dB}$ one way between the generator and the sample and has not been taken into account into the figures 1 and 2 .

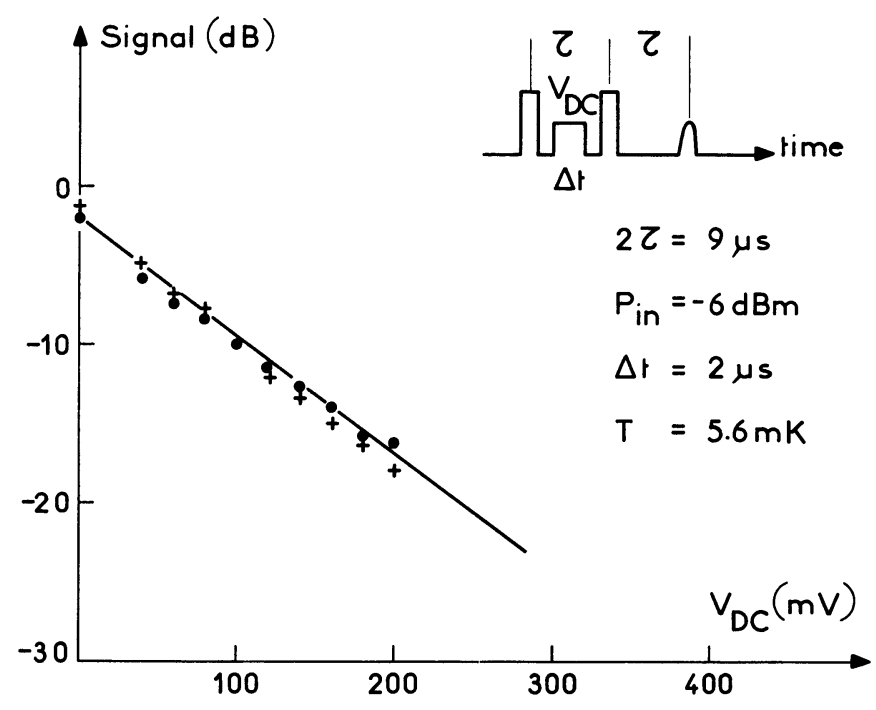

Fig. 2. - The spontaneous signal is plotted as a function of the voltage of the DC pulse. The thickness of the sample is $2 \mathrm{~mm}$.

3. Experimental results. -3.1 The first series of experiments consisted in measuring the signal intensities as a function of the input power $P_{\text {in }}$. A typical curve is given in figure 1 . When we generate the spontaneous echo, both pulses have the same amplitude and we made the width of the second pulse $\left(\Delta t_{2}=0.6 \mu \mathrm{s}\right)$ twice that of the first $\left(\Delta t_{1}=0.3 \mu \mathrm{s}\right)$; they are separated from one another by a time $\tau$ which is a parameter. The curve shown in figure 1 is very typical of what we observe in irradiated quartz. We can distinguish two regimes : at low enough power, there is first an increase of the signal which is proportional to $P_{\text {in }}^{3}$ and then the signal levels off 
and remains constant for more than $12 \mathrm{~dB}$; this is characteristic of a system with widely distributed properties. When the power is further increased, the signal starts to raise again following a $P_{\text {in }}^{3}$ law. It passes through a maximum which gives in fact a very large signal and it starts to decrease once more when it reached the maximum power acceptable for our experimental conditions; acceptable means that for the highest power, we proceed to record the signal using a one shot-analysis in order to avoid any heating of the TLS assembly; in fact, only the last part of the curve is recorded following that procedure which is very time-consuming; it gives the same results as the method used for lower power : box-car sampling or integrator. The same behaviour was observed at different $\tau$ values in two or three pulses sequences.

The maximum at high power is not clearly visible on figure 1; however, many similar curves for different values of $2 \tau$ show that it is there.

If we suppose that, in the material, there are two level systems which have an electrical polarizability (transverse electric dipole moment), we are led to think that there are two kinds of dipoles. At the lowest power by the formula : $E^{\mathrm{II}}=\left(P_{\mathrm{in}}^{\mathrm{II}} Q r\right)^{1 / 2}$ where $r$ is II-dipoles, they have a rather large polarizability; when we just reach the plateau we can write the following formula :

$$
E^{\mathrm{II}} p_{\mathrm{x}}^{\mathrm{II}} \Delta t_{1}=\frac{\pi}{2} \hbar
$$

where $p_{x}^{\text {II }}$ is the transverse electric dipole moment of the species II and $E^{\mathrm{II}}$ is the applied electrical field. The electrical field $E$ in (1) is related to the input power by the formula : $E^{\mathrm{II}}=\left(P_{\text {in }}^{\mathrm{II}} Q r\right)^{1 / 2}$ where $r$ is the characteristic impedance. The estimated value of $p_{\mathbf{x}}^{\text {II }}$ is

$$
p_{\mathrm{x}}^{\mathrm{II}}=1.05 \times 10^{-30} \mathrm{MKS} .
$$

It corresponds to a maximum value of the transverse electric dipole moment on a wide distribution of $p_{\mathbf{x}}^{\text {II }}$ for dipoles of approximately the same resonant frequency.

But the distribution is difficult to infer from similar curves (Fig. 1).

The second distinct maximum is obtained for a power $P_{\mathrm{in}}^{\mathrm{I}}$ and the clear distinction between the two regimes seems to indicate that the species II is quite different from the species I. There also, we can estimate $p_{\mathrm{x}}^{\mathrm{I}}$ by the same formula than (1) and we obtain

$$
p_{\mathrm{x}}^{\mathrm{I}}=5 \times 10^{-32} \mathrm{MKS}
$$

a very small value indeed.

One must be careful not to make confusion with the $p_{z}$ value of the same dipoles measured via the relaxation of the dielectric constant.

3.2 The amplitude of the signal is a measurement of the density of state of the species I or II once their transverse moment is known. The amplitude of the electrical polarization within the sample can be written when the signal has reached its maximum and for the limiting condition $\tau=0$

$$
P^{\mathrm{I}}=n^{\mathrm{I}} \hbar \Delta \omega p_{\mathrm{x}}^{\mathrm{I}} \tanh \left(\frac{\hbar \omega}{2 K T}\right)
$$

where $n^{\mathrm{I}}$ is the density of state per unit volume and unit energy of the TLS, and $\Delta \omega$ the inverse of the pulse width

$$
\Delta \omega=\frac{\pi}{2 \Delta t_{1}}
$$

(that is the bandwidth of the excited electric dipoles provided that the overall bandwidth is much larger); the tanh term is a population factor where $\omega$ is the exciting frequency and $T$ the temperature. Knowing the transverse electric dipole moment, we deduce from the absolute values of the signal $S^{\mathrm{I}}$ and $S^{\mathrm{II}}$ the quantities

$$
\left.\begin{array}{l}
n^{\mathrm{I}}=2 \times 10^{46} \\
n^{\mathrm{II}}=7 \times 10^{43}
\end{array}\right\} \mathrm{MKS} .
$$

The experimental values of the curve of figure 1 have been corrected to take into account the very short $T_{2}$ relaxation time, of both species; in figure 1 it depresses the curve because $\tau$ is longer than $T_{2}$ (see below).

The values given in (6) are a little misleading; they correspond only to the density of state per unit energy in the low frequency limit; nothing can be said about the overall width of defects unless it is precisely explored by similar methods at larger frequencies. However, these values of the density of states must be compared with the value deduced from specific heat measurements. In [4], the value of density of states in the low energy limit is

$$
n_{0}=1.6 \times 10^{46} \mathrm{MKS} \text {. }
$$

The corresponding integrated density of defects was estimated on the basis of a gaussian distribution of the density of state centered on zero energy. It gives

$$
N=1.2 \times 10^{24} \mathrm{~m}^{-3}
$$

$N$ is to be compared with the $\mathrm{Al}$ concentration : $2.4 \times 10^{24} \mathrm{~m}^{-3}$. The width of the gaussian distribution is called the localization temperature. We note that $n_{0}$ is very similar to $n^{\mathrm{I}}$.

3.3 A description of the dynamical properties of the different species I and II would be now in order ; in fact, it is too lengthy to be included in the frame of this letter and we quote here only what is necessary for our final discussion; a detailed discussion with complement data will be published elsewhere.

We have observed that the species II has a very short $T_{1}$ relaxation time, apparently not distributed ; 
$T_{1}^{\mathrm{II}}=50 \mu \mathrm{s}$ at $5.8 \mathrm{mK}$ but this may be due to the relatively low dynamics of the signal. Its temperature variation can be attributed to bottleneck effects : $T_{1}^{\mathrm{II}}$ is inversely proportional to $T^{2}$ [8]: $T_{2}^{\mathrm{II}}$ is corresponding very short $\left(T_{2}^{\mathrm{II}} \simeq 20 \mu \mathrm{s}\right.$ at $\left.T=5.8 \mathrm{mK}\right)$.

Concerning the species I, the $T_{1}$ relaxation time is rather distributed but it includes extremely long relaxation time at these temperatures, so long that they are very difficult to measure; by parenthesis, this obliges us to use a one shot-method to register the curve 1 waiting between two shots a very long time of the order of $10 \mathrm{~min}$. The fact that I entities have so long relaxation time seems to indicate that they are of the same nature than those which have been observed by dielectric measurements on the same crystal [3]. We have observed no indication at all on the possibility of a diffusive process to explain the relaxation of the signal either for I or II defects.

3.4 To better characterize the electric dipole moments involved in these experiments, we would rather prefer to describe the influence of an applied constant electric field or of a pulse of a DC field. That trick was previously used in amorphous systems [9] to explore the distribution of the longitudinal electric dipole moment $p_{\mathbf{z}}$.

In figure 2, we have plotted as a function of the voltage of a DC pulse applied between the first and second impulsion the amplitude of the spontaneous echo for $P_{\text {in }}=P_{\text {in }}^{\text {II }}$ and for a given value of $\tau$.

The fact that the signal rapidly decreases when we apply a rather small electric DC field onto the sample proves that the longitudinal electric dipole of the species II is widely distributed. If the signal is written according to the formula

$$
\text { Signal } \simeq \exp \left(-\frac{2 V}{l} \Delta t \Delta p_{z} \frac{1}{\hbar}\right)
$$

where $V$ is the applied DC field, $\Delta t$ the length in time of the pulse DC field pulse, and $l$ the thickness of the sample.

We find from the curve 2 that the distribution of the component of the permanent dipole moment parallel to the DC field is Lorentzian whose width

$$
\Delta p_{\mathrm{z}}^{\mathrm{II}}=10^{-30} \mathrm{MKS} \text {. }
$$

Once more, we have to specify that that distribution corresponds to those entities who have a well defined frequency or energy and a rather well defined transverse electric moment.

The same experiment was repeated on the entities I. In fact, absolutely no effect was measured even when we applied a pulse DC field as large as $20 \mathrm{~V}$. We concluded

$$
\Delta p_{\mathrm{z}}^{\mathrm{I}}=0 .
$$

This last result may be interpreted in two ways; either the I entities have a very narrow distribution of their longitudinal moment, whatever it is, or it can be also interpreted saying that there is no longitudinal or permanent electric dipole moments for the I dipoles; they can very well have only an induced transverse electric dipole moment.

To try to observe the value of the $p_{\mathrm{z}}$ moment of these dipoles, we thought to apply a constant electric field during the all sequence of pulses in order to sweep the line or the density of states across the frequency of the experiment. In fact, no change at all was observed either for I or II dipoles when we increased the constant electric field up to $1.5 \mathrm{kV} / \mathrm{cm}$. Once more, there are two interpretations : if there is no permanent electric dipole moment, we expect no effect at all, it would fit the result observed by applying a DC field on I dipoles; but also, it can be said, in particular for the II dipoles that the density of state is wider than the product of a value of $p_{z}$ times the constant electric field applied ; an experiment performed by a simple resonance method has proved [2] at a higher frequency that in fact, to sweep the line, it was necessary to apply at least $10 \mathrm{kV} / \mathrm{cm}$. We are rather far from these values and then, there is no contradiction with our results.

4. Interpretation. - The question now is : Can we identify the different centres that we observe with what is already known from dielectric or thermal conductivity or specific heat measurements ? Generally, the opinion has been established that these centres are associated with the presence of small quantities of aluminium ions substituted on the $\mathrm{Si}$ site, the surplus of negative charges being compensated for by positive alcali ions somewhere else in the crystal. The $\gamma$ irradiation thus causes two centres : holes trapped at the neighbouring oxygen ions and an alcali ion associated with a trapped electron somewhere else.

In our opinion, many experimental facts, including our measurements, seem to show that the aluminium sites correspond to the I species that we are observing (in the notation of Stevels and Volger they are noted $\left.{ }^{\mathrm{Al}} \mathrm{A}_{+}\right)$; these centres have a rather long $T_{1}$ relaxation time; we have noticed that it is even very difficult to measure it in the mK range; thus they could be assimilated to those which are responsible for the dielectric losses in the audio frequency range at helium temperature; their density of state is relatively large at low energy and their distribution in energy can be described in terms of a localization temperature [3] which was previously measured to be of the order of a few $\mathrm{K}$. The experimental value of the density of state given by (6) is in agreement with the dielectric constant measurements. As well, we have also recognized that the distribution of their longitudinal electric dipole moment is very narrow; this is in agreement with the crystallinity of quartz which suggests a preferential orientation of the axis of the dipole; that would reinforce the idea that a hole is 
located between two oxygen ions making a defined angle with the $C$ axis of the crystal.

Concerning the electron centres which are called ${ }^{\mathrm{x}} p_{-}$centres by Stevels and Volger, we have a tendency to identify them with the II species that we observe for II-dipoles. It is also quite natural that in this case, the density of states $n^{\text {II }}$ given by (6) should be much smaller, corresponding to a broader distribution of energy levels. It has been suggested as well that in a quartz with a rather high degree of perfection there are less electron centres than hole centres because some of the electron centres correspond to associated entities : they form clusters; this would favour in fact the high polarizability that we observe for the II centres ( $p_{\mathrm{x}}^{\mathrm{II}}$ large); $n^{\text {II }}$ could then be smaller than $n^{\mathrm{I}}$. These II centres, as we have observed, have a rather short relaxation time or a rather large coupling constant with the lattice and the phonons ; in principle, we should have observed a decrease of the thermal conductivity due to the scattering of phonons by these centres. In fact, a new thermal conductivity measurement [10] invokes clusters whose size should be of the order of the wavelength of the dominant phonons to have an effect; this may very well be possible : when we make a calculation of the heat conductivity based on the density of state $n^{\mathrm{II}}$, and on the coupling with the lattice, it appears that there is no net decrease of the thermal conductivity by resonant scattering, mainly because the density of state is quite small.

Concerning the irradiated quartz crystal, we are thus led to think that there are two distinct centres which we have identified first with holes tunneling between two oxygen atoms; these are our I centres whose density of state is as large as the one obtained in specific heat measurements ; they are poorly coupled to the lattice and responsible for dielectric anomalies at low temperature; they are non-associated defects. The second centres are imagined as clusters with large electrical polarizabilities ; our II-centres, strongly coupled with the lattice and with widely distributed properties.

As a conclusion, we would like to draw attention on a comparison between irradiated quartz and silica glass [11]. At first sight, the distinction between the two is quite sharp : in silica, there is no clear difference between two distinct entities, but rather a broad distribution of properties; the active centres are wellknown to be strongly coupled to the lattice and govern most of the thermal properties; the dielectric properties are markedly dependant of the $\mathrm{OH}^{-}$ions imbeded in the glass. However, the question can be raised : even if the dielectric properties are apparently related with the $\mathrm{OH}^{-}$content, are there some other intrinsic defects which could generate electronic centre with a certain polarizability even in a pure glass? These centres would be responsible of the intrinsic properties of the glass and their nature related with the structural disorder of the glassy state. The two level systems (TLS) in the glass would then be of electronic character as well as the I-centre in quartz.

\section{References}

[1] Taylor, A., Farnell, G., Canad. J. Physics 42 (1964) 594.

[2] Kerssen, J., Volger, J., Phys. Lett. 24A (1967) 657.

[3] De Vos, W., Volger, J., Physica 47 (1970) 13.

[4] Saint-Paul, M., Picot, B., Nava, R., Phys. Lett. 66A (1978) 389.

[5] Saint-Paul, M., Nava, R., J. Physique 39 (1978) 786.

[6] Chaussy, J., Gilchrist, J., Lasjaunias, J., Saint-Paul, M., Nava, R., J. Phys. Chem. Solids (to be published)

[7] Bernard, L., Piche, L., Schumacher, G., Joffrin, J., Graebner, J., J. Physique-Lett. 39 (1978) L-126.
[8] Abragam, A. and Bleaney, B., Electron paramagnetic resonance of transition ions (Oxford) 1970.

[9] Bernard, L., Piche, L., Schumacher, G., Joffrin, J., J. Physique Colloq. 39 (1978) C6-957.

[10] Wasim, S., Nava, R., Phys. Status Solidi (a) 51 (1979) 359.

[11] Bernard, L., Piche, L., Schumacher, G., Joffrin, J., J. Low Temp. Phys. 35 (1979) 411.

[12] Natural Brazilian quartz crystal supplied by Valpey-Fisher corp. (Hopkinton Mass., U.S.A.). 